\title{
Bericht der Department Editors der ZfB
}

Die Zeitschrift für Betriebswirtschaft veröffentlicht methodisch und inhaltlich innovative, wissenschaftliche Beiträge zu theoretischen Entwicklungen und praktischen Anwendungen aus allen Gebieten der Betriebswirtschaftslehre. Zur Gewährleistung des qualitativen Standards werden die bei der ZfB eingereichten Beiträge einem doppelt verdeckten Begutachtungsverfahren mit zwei Fachgutachtern unterzogen. Bei abweichenden Gutachten wird ein Drittgutachter bestellt. Die Betreuung der eingegangenen Manuskripte erfolgt durch jeweils einen der vierzehn Department Editors. Sie setzen den Prozess der Begutachtung in Gang und entscheiden schließlich über die Annahme oder Ablehnung eines Beitrags. Der Prozess des Manuskriptmanagements wird durch den Editor-in-Chief koordiniert. Er nimmt die inhaltliche Gestaltung der Hefte vor.

Im Verlauf des Jahres 2006 sind bei der ZfB 141 neue Beiträge eingegangen. 67 Manuskripte befanden sich bereits am Anfang des Zeitraums in der Begutachtung. Von diesen insgesamt 208 Beiträgen wurden im Jahre 2006 143, das entspricht 69\%, einer Herausgeberentscheidung zugeführt. 43 Manuskripte oder 30\% wurden zur Veröffentlichung angenommen.

Die Sicherstellung der Veröffentlichung qualitativ hochwertiger Forschungsergebnisse und Praxisberichte durch die ZfB hängt wesentlich von der Unterstützung durch das Editorial-Board sowie einer Vielzahl hervorragender Gutachter ab. Im Anschluss veröffentlichen wir deshalb die Liste der Gutachter, die im Referenzzeitraum mindestens eine Begutachtung für die ZfB abgeschlossen haben. Ihnen gilt unser besonderer Dank. Nur durch die engagierte Mitarbeit der externen Gutachter ist sichergestellt, dass die ZfB in der zurückliegenden Berichtsperiode die ganze Breite betriebswirtschaftlicher Forschung repräsentieren kann.

\section{Die Gutachter}

Adler, Jost, Duisburg

Ahlert, Dieter, Münster

Ahrens, Joachim, Göttingen

Albach, Horst, Bonn

Albers, Sönke, Kiel

Alewell, Dorothea, Jena

Altenburger, Otto, Wien

Baier, Daniel, Cottbus

Bannier, Christina, Frankfurt am Main

Barzantny, Cordula, Toulouse

Bauer, Hans H., Mannheim

Bauer, Thomas, Bochum

Becker, Fred, Bielefeld

Berendt, Bettina, Berlin

Bertschek, Irene, Mannheim
Bilstein, Frank, Bonn

Blaga, Steffen, Hagen

Böcking, Hans-Joachim, Frankfurt am Main

Braun, Thomas, Bielefeld

Braun, Sebastian, Paderborn

Breithecker, Volker, Duisburg

Bresser, Rudi, Berlin

Brettel, Malte, Aachen

Bruhn, Manfred, Basel

Bühner, Rolf, Passau

Casey, Christopher, Wien

Chwolka, Anne, Magdeburg

Clement, Michel, Hamburg

Dibbern, Jens, Mannheim

Dierkes, Stefan, Marburg 
Dietl, Helmut, Zürich

Dilger, Alexander, Münster

Diller, Hermann, Nürnberg

Dittmann, Ingolf, Rotterdam

Djanani, Christiana, Ingolstadt

Dowling, Michael, Regensburg

Drobetz, Wolfgang, Basel

Dyckhoff, Harald, Aachen

Eggert, Andreas, Paderborn

Eiben, Jan, Vallendar

Eisend, Martin, Berlin

Fallgatter, Michael, Wuppertal

Fandel, Günter, Hagen

Fassnacht, Martin, Vallendar

Fassott, Georg, Kaiserslautern

Federrath, Hannes, Regensburg

Fiedler, Marina, München

Fink, Andreas, Hamburg

Fischer, Marc, Kiel

Föhr, Silvia, Leipzig

Förster, Guido, Düsseldorf

Fraberger, Friedrich, Wien

Franck, Egon, Zürich

Frank, Ulrich, Essen

Franke, Nikolaus, Wien

Fransoo, Jan, Eindhoven

Freidank, Carl-Christian, Hamburg

Frick, Bernd, Witten

Fülbier, Rolf Uwe, Vallendar

Gassen, Joachim, Berlin

Gaus, Hansjörg, Chemnitz

Gemünden, Hans-Georg, Berlin

Gerpott, Torsten, Duisburg

Gmür, Markus, Oestrich-Winkel

Göbel, Stefan, Rostock

Götze, Uwe, Chemnitz

Gräfer, Horst, Paderborn

Grichnik, Dietmar, Witten

Gröppel-Klein, Andrea, Saarbrücken

Gruber, Marc, Lausanne

Grund, Christian, Würzburg

Grunert, Jens, Tübingen

Gugler, Klaus, Wien

Günther, Hans-Otto, Berlin

Gürtler, Marc, Braunschweig
Haase, Michaela, Berlin

Hachmeister, Dirk, Stuttgart

Hack, Andreas, Dortmund

Hamerle, Alfred, Regensburg

Harhoff, Dietmar, München

Helmig, Bernd, Fribourg

Henkel, Joachim, München

Herrmann, Andreas, St. Gallen

Heyd, Reinhard, Schwäbisch Gmünd

Hoeck, Michael, Hamburg

Hoffjan, Andreas, Vallendar

Hofmann, Christian, Tübingen

Högl, Martin, Vallendar

Holzmüller, Hartmut, Dortmund

Homburg, Carsten, Köln

Homburg, Christian, Mannheim

Hruschka, Harald, Regensburg

Huber, Frank, Mainz

Huschens, Stefan, Dresden

Hutzschenreuter, Thomas, Vallendar

Jensen, Ove, Mannheim

Johanning, Lutz, Oestrich-Winkel

Jost, Peter J., Vallendar

Jungwith, Carola, Vaduz

Kaas, Klaus Peter, Frankfurt am Main

Kahle, Holger, Stuttgart-Hohenheim

Kajüter, Peter, Münster

Kappler, Ekkehard, Innsbruck

Kaserer, Christoph, München

Kasperzak, Rainer, Berlin

Kempf, Alexander, Köln

Kersten, Gregory E., Montreal

Kessler, Wolfgang, Freiburg im Breisgau

Kieser, Alfred, Mannheim

Kiesewetter, Dirk, Magdeburg

Klodt, Henning, Kiel

Klos, Alexander, Münster

Knoll, Leonard, Würzburg

Knolmayer, Gerhard, Bern

König, Rolf, Bielefeld

König, Wolfgang, Frankfurt am Main

Königstein, Manfred, Erfurt

Konrad, Elmar, Dortmund

Koschate, Nicole, Nürnberg

Kotzab, Herbert, Frederiksberg 
Kraft, Gerhard, Halle (Saale)

Kräkel, Matthias, Bonn

Krapp, Michael, Augsburg

Krawitz, Norbert, Siegen

Krcmar, Helmut, Garching b. München

Kreikebaum, Hartmut, Oestrich-Winkel

Krohmer, Harley, Bern

Kruschwitz, Lutz, Berlin

Kuckertz, Andreas, Essen

Kuhn, Heinrich, Ingolstadt

Kuhner, Christoph, Köln

Kundisch, Dennis, Freiburg

Küpper, Hans-Ulrich, München

Kupsch, Peter, Bamberg

Küting, Karlheinz, Saarbrücken

Kutschker, Michael, Ingolstadt

Laitenberger, Jörg, Hannover

Langer, Thomas, Münster

Lehmann, Matthias, Trier

Lehmann, Erik E., Augsburg

Leibfried, Peter, St. Gallen

Lenz, Hansrudi, Würzburg

Letmathe, Peter, Siegen

Linde, Rainer, Jena

Locarek-Junge, Hermann, Dresden

Löffler, Andreas, Nürnberg

Luptácik, Mikulás, Wien

Mager, Ferdinand, Nürnberg

Mahayni, Antje, Duisburg

Mandl, Gerwald, Graz

Mann, Andreas, Kassel

Matiaske, Wenzel, Flensburg

Mayer, Horst, Dresden

Mellewigt, Thomas, Berlin

Mellis, Werner, Köln

Mellwig, Winfried, Frankfurt am Main

Merz, Christin, Vallendar

Minner, Stefan, Mannheim

Mochty, Ludwig, Essen

Möhrle, Martin G., Bremen

Möller, Hans-Peter, Aachen

Mölls, Sascha, Marburg

Muck, Matthias, Bamberg

Müller, Sigrid, Berlin

Neumann, Gustaf, Wien
Neus, Werner, Tübingen

Nicolai, Alexander, Oldenburg

Nietert, Bernhard, Marburg

Nippel, Peter, Kiel

Nöth, Markus, Hamburg

Olbrich, Michael, Trier

Ott, Robert, Regensburg

Otto, Andreas, Regensburg

Paulssen, Marcel, Berlin

Pfeiffer, Thomas, Wien

Picot, Arnold, München

Piller, Frank T., Aachen

Poddig, Thorsten, Bremen

Post, Thomas, Berlin

Pummerer, Erich, Innsbruck

Reese, Joachim, Lüneburg

Rettberg, Jan F., Dortmund

Richter, Frank, Ulm

Riesenhuber, Felix, Kiel

Röder, Klaus, Regensburg

Rogler, Silvia, Freiberg

Rösch, Daniel, Regensburg

Rück, Hans R. G., Worms

Rückle, Dieter, Trier

Rudolf, Markus, Vallendar

Rudolph, Bernd, München

Ruhnke, Klaus, Berlin

Sackmann, Sonja, Neubiberg

Sadowski, Dieter, Trier

Salomo, Sören, Graz

Sander, Matthias, Konstanz

Schade, Christian, Berlin

Schauenberg, Bernd, Freiburg

Schertler, Andrea, Kiel

Schiereck, Dirk, Oestrich-Winkel

Schiller, Ulf, Bern

Schlüchtermann, Jörg, Bayreuth

Schmeiser, Hato, St. Gallen

Schmidt, Matthias, Leipzig

Schmidt, Klaus, München

Schmidt, Axel, Trier

Schneeloch, Dieter, Hagen

Schoder, Detlef, Köln

Schrader, Ulf, Hannover

Schreiber, Ulrich, Mannheim 
Schreyögg, Georg, Berlin

Schruff, Lothar, Göttingen

Schuhmacher, Frank, Leipzig

Schüler, Andreas, Neubiberg

Schultze, Wolfgang, Jena

Schwaiger, Manfred, München

Schwalbach, Joachim, Berlin

Schwerk, Anja, Berlin

Schwetzler, Bernhard, Leipzig

Siegel, Theodor, Berlin

Sigloch, Jochen, Bayreuth

Simon, Hermann, Bonn

Simon, Patricia, Würzburg

Simons, Dirk, Mannheim

Sinz, Elmar J., Bamberg

Skiera, Bernd, Frankfurt am Main

Sliwka, Dirk, Köln

Souren, Rainer, Ilmenau

Spann, Martin, Passau

Speckbacher, Gerhard, Wien

Spengler, Thomas, Magdeburg

Spremann, Klaus, St. Gallen

Stadtmann, Georg, Vallendar

Starck, Markus, Mainz

Steffenhagen, Hartwig, Aachen

Stehle, Richard, Berlin

Steiner, Manfred, Augsburg

Steiner, Peter, Graz

Stock-Homburg, Ruth, Darmstadt

Stokburger-Sauer, Nicola, Mannheim

Stolper, Markus, Dortmund

Stotz, Olaf, Aachen

Strassberger, Mario, Jena

Streim, Hannes, Bochum

Streitferdt, Felix, Trier

Sürie, Christopher, Darmstadt

Swoboda, Bernhard, Trier

Sydow, Jörg, Berlin

Teichert, Thorsten, Hamburg

Temme, Dirk, Berlin

Theile, Carsten, Bochum

Theisen, Manuel René, München

Theissen, Eric, Bonn

Tiechert, Thorsten, Hamburg
Trautmann, Siegfried, Mainz

Trommsdorf, Volker, Berlin

Troßmann, Ernst, Stuttgart

Tscheulin, Dieter, Freiburg im Breisgau

Tuschke, Anja, Bern

van den Poel, Dirk, Ghent

Velthuis, Louis, Frankfurt am Main

Vera, Antonio, Köln

Voeth, Markus, Stuttgart

Vogt, Bodo, Magdeburg

von Ahsen, Anette, Essen

von der Oelsnitz, Dietrich, Ilmenau

von Portatius, Hans Botho, Bonn

von Wangenheim, Florian, München

Voß, Stefan, Hamburg

Wagner, Dieter, Potsdam

Wagner, Franz, Tübingen

Wagner, Udo, Wien

Wagner, Niklas, Passau

Wallau, Frank, Paderborn

Warning, Susanne, Trier

Watrin, Christoph, Münster

Wehrheim, Michael, Marburg

Weichenrieder, Alfons, Frankfurt

Weigand, Jürgen, Vallendar

Weißenberger, Barbara E., Gießen

Weitzel, Tim, Bamberg

Weller, Ingo, Berlin

Wellisch, Dietmar, Hamburg

Wendt, Oliver, Kaiserslautern

Wielenberg, Stefan, Hannover

Wiese, Harald, Leipzig

Wilhelm, Jochen, Passau

Wilkens, Marco, Ingolstadt

Wimmer, Frank, Bamberg

Witt, Peter, Dortmund

Wolf, Joachim, Kiel

Wolff, Birgitta, Magdeburg

Woratschek, Herbert, Bayreuth

Wüstemann, Jens, Mannheim

Zelewski, Stephan, Essen

Zimmermann, Jochen, Bremen

Zimmermann, Jürgen, Clausthal-Zellerfeld

Zwick, Thomas, Mannheim 


\section{Editorial}

Die vorliegende Ausgabe als Doppelheft sollte zwar dazu dienen, den Überschuss der bereits publizierten Heftseiten dieses Jahrgangs mit dem geplanten Umfang abzugleichen, dennoch enthält dieses Heft vier Beiträge, von denen einer als Übersichtsartikel vergleichsweise lang ausfällt. Die angestrebte thematische Mischung der abgedruckten Manuskripte empfahl jedoch die Gestaltung des Heftes in der Weise, wie Sie es nun in den Händen halten. Die Beiträge dieses Heftes kommen aus den Bereichen Banken, Controlling, Produktion und Unternehmensführung.

\section{Einflussfaktoren der Recovery Rate von Bankkrediten}

Auf der Grundlage einer Umfrage bei fast 1400 Volks- und Raiffeisenbanken in Deutschland im Jahre 2004, bei der 428 Rückantworten ausgewertet werden konnten, untersuchen Grunert und Weber mithilfe statistischer Methoden die Bedeutung potentieller Einflussfaktoren auf die Höhe der Recovery Rate von Bankkrediten an kleine und mittlere Unternehmen. Die Ergebnisse der empirischen Untersuchung sind unmittelbar für die Steuerung der Kreditportfolios beziehungsweise Kreditrisiken sowie die Zinskalkulation in der Bankpraxis verwertbar. Als Einflussfaktoren auf die Recovery Rate analysieren die Autoren die Eigenkapitalquote, die Besicherungsquote, den Rang der Ansprüche beim Kreditnehmer sowie die Unternehmensgröße, Branchenzugehörigkeit des Kreditnehmers wie auch die Ausfallwahrscheinlichkeit des Kredits, die makroökonomischen Verhältnisse und die Länge der Geschäftsbeziehungen zwischen Kreditnehmer und Bankinstitut. Die Recovery Rate entspricht dabei der Wiedergewinnungsquote auf den verbleibenden Kreditbetrag bei Ausfall des Kreditnehmers. Diese ergibt sich aus Einzahlungen infolge der Verwertung von Kreditsicherheiten, aus Auszahlungen aufgrund von Mitarbeitergehältern der Bank und der Bemühung von Rechtsbeistand und hängt vom Ausfallereignis ab, das durch Insolvenzantrag oder Zahlungsverzug definiert sein kann. Um die Bedeutung potentieller Einflussfaktoren zu bestimmen, werden zwei unterschiedliche Befragungstechniken angewendet. Zunächst wird die Bedeutung ermittelt, welche die Befragten den Faktoren direkt beimessen, wenn sie von ihnen jeweils einzeln einzuschätzen sind. Danach erfolgt eine kombinierte Abfrage auf der Grundlage einer Conjoint-Analyse, die offenbaren soll, inwieweit die Antworten in Abhängigkeit der Befragungstechniken unterschiedlich ausfallen. Der Vergleich der Befragungsergebnisse ermöglicht es zugleich, die Antworten auf Konsistenz zu prüfen und durch statistische Auswertungen herauszufinden, inwieweit bedeutenden Faktoren innerhalb beider Befragungstechniken auch tatsächlich immer ein größerer Einfluss zugemessen wird. Die Ergebnisse zeigen, dass in beiden Befragungstechniken der Eigenkapitalquote, der Besicherungsquote und der Ausfallwahrscheinlichkeit eine hohe Bedeutung im Hinblick auf die Höhe der Recovery Rate zukommt. Die Conjoint-Analyse auf der Grundlage der kombinierten Befragung ergibt zudem, dass die makroökonomischen Verhältnisse ebenfalls die Höhe der Recovery Rate beeinflussen. Die Unternehmensgröße und die Branchenzugehörigkeit spielen dagegen kaum eine Rolle. In diesem Zusammenhang ist die ermittelte negative Korrelation zwischen der Ausfallwahrscheinlichkeit und der Recovery Rate von besonderem praktischen 
und theoretischen Interesse. Modelltheoretische Betrachtungen unterstellen in der Regel eine Unabhängigkeit beider Größen, und die negative Korrelation bedeutet praktisch, dass seitens der Banken bei der Kreditvergabe eine Risikounterschätzung stattfindet.

\section{Controlling und Operations Research}

Küpper verfolgt in seinem Beitrag das Anliegen, die Bezüge zwischen dem Controlling und dem Operations Research herauszuarbeiten und zu zeigen, wie sich aus der praxisorientierten Disziplin des Controlling unter Nutzung von quantitativen Ansätzen des Operations Research allmählich ein wissenschaftliches Gebiet der Betriebswirtschaftslehre herausgebildet hat, welches zu praktisch relevanten Erkenntnissen führt und Anregungen zu eigenständigen Forschungsperspektiven gibt. Zur Erhellung der Hintergründe dieser Bezüge werden zunächst die Parallelen und Unterschiede in den Entwicklungen von Controlling und Operations Research zu Teildisziplinen der Betriebswirtschaftslehre dargelegt. Der Autor führt aus, dass sich das Controlling erst in den letzten 15 Jahren zu einem Forschungs- und Lehrgebiet der Betriebswirtschaftlehre herausgebildet hat und dabei insbesondere die innerbetriebliche Koordination und Steuerung eine Kernfunktion des Controlling ist, zu deren Bewältigung das Operations Research wertvolle Beiträge leisten kann. Während jedoch die Entwicklung des Operations Research in Deutschland nach dem 2. Weltkrieg schnell voranschritt und sich diese Disziplin in Forschung und Lehre an den Universitäten sowie in den Planungsabteilungen großer Unternehmungen und Institutionen rasch etablierte, war die Entwicklung des Controlling in Deutschland während dieser Zeit eher zögerlich, da lange Zeit keine Klarheit über den Inhalt dieses neuen Wissenschaftsgebietes herrschte. So führt Küpper aus, dass das Controlling schließlich seine theoretische Fundierung im Wesentlichen durch drei Theorieansätze erfahren hat, welche die Aufgabe der innerbetrieblichen Koordination und Steuerung in leistungsfähiger Weise unterstützen: nämlich durch Optimierungsmodelle, welche zur Lösung von Ansätzen der simultanen und hierarchischen Planung beitrugen, durch Ansätze der Informationsökonomie, durch welche die Beschaffung, Verarbeitung und Generierung von Daten bewerkstelligt wurde, und schließlich durch die Agency-Theorie, wodurch die erfolgsorientierte Delegierung von Entscheidungen modelliert werden konnte.

\section{Produktionsplanung bei Variantenfließfertigung}

Boysen, Fliedner und Scholl erörtern in ihrem Beitrag die Planungshierarchie und die Elemente einer hierarchischen Planung für die Variantenfließfertigung, bei der unterschiedliche Varianten eines Grundmodells gemeinsam auf einem Fließsystem in wahlfreier Reihenfolge gefertigt werden, wie dies beispielsweise bei der Endmontage der Automobilindustrie und der Elektroindustrie der Fall ist. Die kundenindividuelle Anpassung der Produkte im Rahmen einer effizienten Fließfertigung bildet den Hintergrund der angestellten Überlegungen. Die Autoren weisen darauf hin, dass Betrachtungen zur Variantenfließfertigung bisher entweder unter dem lang- beziehungsweise mittelfristigen Aspekt der Fließbandabstimmung oder aber unter dem kurzfristigen Aspekt der Reihenfolgeplanung durch- 


\section{Editorial}

geführt worden sind. Zwischen diesen beiden Anfangs- und Endpunkten der Planungshierarchie ist jedoch im Allgemeinen die Bestimmung des Fertigungsprogramms als Zwischenschritt einzubetten. Eine Integration der Problemstellung innerhalb eines einheitlichen Ansatzes ist bisher in der Literatur kaum vorzufinden. So diskutieren die Autoren als Elemente eines hierarchischen Planungssystems für die Produktionsform der Variantenfließfertigung die Erstinstallation der Fließlinie, die Produktionsprogrammplanung, die Rekonfiguration des Fließsystems, die Reihenfolgeplanung sowie das Resequencing. Diese Elemente dienen dazu, den konzeptionellen Rahmen eines Produktionsplanungssystems für Variantenfließfertigung zu entwickeln und dabei die Ein-Produkt-Fließbandabstimmung auf den Fall variantenreicher Fertigung zu erweitern. Die dargestellten Elemente der hierarchischen Planung für eine Variantenfließfertigung lassen zugleich erkennen, worin die noch offenen Forschungsfragen für eine praxistaugliche hierarchische Planung liegen.

\section{Überblick über die empirische Change Management-Forschung}

Stock-Homburg gibt in ihrem Beitrag einen Überblick über 16 Jahre empirischer Change Management-Forschung in den Jahren 1990 bis 2006. In den Überblick sind 137 empirische Arbeiten einbezogen, die sowohl quantitative als auch qualitative Studien enthalten, sich auf die Makroebene der Unternehmensorganisation oder die Mikroebene des Teams oder der Einzelperson eines Unternehmens erstrecken und in führenden nationalen und internationalen englischsprachigen Zeitschriften erschienen sind. Unternehmensveränderungen können sich in der Veränderung von Strategien sowie der Kultur und der Struktur des Unternehmens ausdrücken, aber auch durch Veränderungen in den Einstellungen und Verhaltensweisen der Unternehmensmitglieder bedingt sein. Auslöser für derartige Unternehmensveränderungen sind der zunehmende Wettbewerb, veränderte Kundenanforderungen, technologische Entwicklungen, die Internationalisierung von Unternehmungen, gesetzliche Veränderungen oder der Wechsel in der Geschäftsführung. Da die Abstände tief greifender Veränderungen in Unternehmungen zunehmend kürzer werden, stellt sich die Frage, wie man diese Unternehmensveränderungen mithilfe des Change Managements erfolgreich gestalten kann. Bei der überblicksmäßigen Analyse der empirischen Veröffentlichungen stehen Auslöser sowie Intensität und Umfang der Unternehmensveränderungen als auch die jeweilige Unternehmensebene, auf der diese Veränderungen stattfinden, im Mittelpunkt der Betrachtungen. Die Ergebnisse werden in tabellarischen Übersichten zusammengetragen, welche die Veröffentlichung, den Gegenstand der Untersuchung sowie die zugrunde liegende Datenbasis aufführen. Zum Schluss stellt die Autorin die Implikationen zusammen, die sich nach ihrer Erkenntnis aus den bisherigen Ergebnissen für die zukünftige Forschung ergeben.

\section{Das September-Heft}

Das nächste Heft wird drei Beiträge enthalten, von denen einer wiederum ein Übersichtsaufsatz ist. Die Arbeiten kommen aus den Gebieten des Controlling, des Kommunikationsmanagements sowie der Rechnungslegung. 
Homburg und Wrede untersuchen in ihrer Arbeit, inwieweit Gläubiger von kleinen und mittelgroßen Unternehmen den Cash Flow mithilfe aktueller Jahresabschlussgrößen einschätzen können. Hierzu werden unterschiedliche Erklärungsmodelle formuliert und auf der Grundlage eines umfänglichen Datenpanels empirisch analysiert. Dabei geht es darum, persistente Bestimmungsgrößen des künftigen operativen Cash Flows herauszufiltern.

Die Erfolgswirkung des internetbasierten Supply Chain Managements untersuchen Wirtz und Wecker. Sie versuchen die Frage zu klären, ob der Einsatz von Internettechnologien im Supply Chain Management einen kritischen Erfolgsfaktor darstellt. Zu diesem Zweck werden die wesentlichen Determinanten des internetbasierten Supply Chain Management-Modells konzeptionalisiert und operationalisiert sowie entsprechende Hypothesen formuliert. Die empirische Überprüfung der Hypothesen stützt sich auf eine branchenübergreifende schriftliche Befragung, bei der 350 Rückantworten ausgewertet werden konnten.

Mölls und Strauß versuchen durch ihre Übersichtsarbeit eine Antwort auf die Frage zu finden, inwieweit die Informationen der Rechnungslegung für die Unternehmensbewertung relevant sind, indem sie Stand und Implikationen der empirischen Forschung für Aktionäre und Regulierer systematisch aufbereiten. Dabei decken sie zugleich Defizite im gegenwärtigen Stand der empirischen Forschung auf.

\section{Günter Fandel}

Editor-in-Chief 